\title{
On Numerical relativistic hydrodynamics and barotropic equations of state
}

\author{
José María Ibáñez ${ }^{1}$, Isabel Cordero-Carrión ${ }^{2}$ and Juan Antonio \\ Miralles ${ }^{3}$ \\ ${ }^{1}$ Department of Astronomy and Astrophysics, University of Valencia, E-46100, \\ Burjassot, Spain \\ 2 Max-Planck-Institute for Astrophysics, Garching, Karl-Schwarzschild-Str. 1, \\ D-85741, Garching, Germany \\ ${ }^{3}$ Department of Applied Physics, University of Alicante, Campus de Sant Vicent del \\ Raspeig, E-03080, Alicante, Spain \\ E-mail: jose.m.ibanez@uv.es, chabela@mpa-garching.mpg.de, \\ ja.miralles@ua.es
}

\begin{abstract}
The characteristic formulation of the relativistic hydrodynamic equations (Donat et al 1998 J. Comput. Phys. 146 58), which has been implemented in many relativistic hydro-codes that make use of Godunov-type methods, has to be slightly modified in the case of evolving barotropic flows. For a barotropic equation of state, a removable singularity appears in one of the eigenvectors. The singularity can be avoided by means of a simple renormalization which makes the system of eigenvectors well defined and complete. An alternative strategy for the particular case of barotropic flows is discussed.

PACS numbers: 04.25.D-, 47.11.-j, 47.75.+f, 95.30.Sf
\end{abstract}


Barotropic fluids obeying an equation of state (EoS) of the type $p=p(e)$ (barotropic EoS), where $e=\rho(1+\epsilon)$ is the total mass-energy density ( $\rho$ and $\epsilon$ being, respectively, the rest-mass density and the internal specific energy), are a particular class of fluids which become familiar, for example, to cosmologists interested in the analysis of linear perturbations of radiation-dominated universes (see, e.g., [2]), or to astrophysicists interested in the physics of dense matter where the asymptotic EoS for high density and cold matter is barotropic (see, e.g., 3]). The mathematical properties of relativistic Euler equations for barotropic EoS have been studied in [4]. A particular case of a barotropic EoS is the ultrarelativistic EoS, where pressure is directly proportional to the energy density. Recently, several authors have paid particular attention to this ultrarelativistic EoS in order to find the exact solution of the relativistic hydrodynamical Riemann problem with nonzero tangential velocities [5], the corrugation instabilities occurring for solutions of that Riemann problem [6], or just in order to compare efficiencies of relativistic hydro-codes specifically designed for such particular EoS [7].

Twenty years ago, authors in [8] paved the way for modern numerical relativistic hydrodynamics (RHD) extending to this field the strategy of using high-resolution shockcapturing (HRSC) methods. By exploiting the hyperbolic and conservative characters of the RHD equations, these authors (and collaborators) derived the full spectral decomposition of the Jacobian matrices associated with the fluxes in a series of papers: [8], in 1D (one-dimensional) general relativity hydrodynamics (GRHD); 9] and [1], in multidimensional special relativity hydrodynamics (SRHD); 10] (where the eigenvectors were given for a diagonal metric) and [11] (where authors derived the full spectral decomposition: right and left eigenvectors) in multidimensional GRHD. This numerical strategy has proved to be very fruitful in the field of computational RHD (see, e.g., the reviews 12 and 13 ).

All the above papers (and works related with them) as well as all the current numerical codes based on these references make use of a general EoS of the form $p=p(\rho, \epsilon)$. This general EoS contains some particular cases (see, e.g., [14], [15], [16]): (i) dust $(p=0)$. For this EoS, motion equations can be reduced to the inviscid Burger's equation (in 1D). (ii) A gamma-law $\operatorname{EoS}\left(p=K \rho^{\gamma}\right)$. This case makes one of the motion equations (the energy equation) redundant.

This note is addressed to those researchers, working in relativistic astrophysics or numerical relativity, that might be interested in studying the evolution of barotropic fluids by using relativistic hydro-codes originally designed to use Godunov-type methods and a general EoS, of the type $p=p(\rho, \epsilon)$.

The evolution of a relativistic fluid is described by a system of equations which are the expression of local conservation laws: the local conservation of baryon number and energy-momentum, respectively,

$$
\begin{aligned}
& \nabla_{\mu} J^{\mu}=0, \\
& \nabla_{\mu} T^{\mu \nu}=0,
\end{aligned}
$$


the current $J^{\mu}$ and the energy-momentum tensor $T^{\mu \nu}$ being

$$
\begin{aligned}
& J^{\mu}=\rho u^{\mu}, \\
& T_{\mu \nu}=\rho h u_{\mu} u_{\nu}+p g_{\mu \nu} .
\end{aligned}
$$

(Greek and Latin indices run, respectively, from 0 to 3 and from 1 to 3. In the units we use, the speed of light is equal to one).

In the above equations, $h$ is the specific enthalpy, defined by $h=1+\varepsilon+p / \rho, u^{\mu}$ is the 4 -velocity of the fluid, and $g_{\mu \nu}$ defines the metric of a general spacetime $\mathcal{M}$ where the fluid evolves, $\nabla_{\mu}$ stands for the covariant derivative.

In the following we will restrict our analysis to the Minkowski spacetime. The above system of equations, (11) and (2), can be written as:

$$
\frac{\partial \mathbf{F}^{0}(\mathbf{w})}{\partial t}+\frac{\partial \mathbf{F}^{i}(\mathbf{w})}{\partial x^{i}}=\frac{\partial \mathbf{F}^{0}(\mathbf{w})}{\partial t}+B^{i} \frac{\partial \mathbf{F}^{0}(\mathbf{w})}{\partial x^{i}}=0
$$

where

$$
B^{i}=\frac{\partial \mathbf{F}^{i}(\mathbf{w})}{\partial \mathbf{F}^{0}(\mathbf{w})}
$$

are three $5 \times 5$ Jacobian matrices and $\mathbf{w}=\left(\rho, v^{j}, \varepsilon\right)$ is the 5 -vector of primitive variables. The components of the 3-velocity, $v^{j}$, are defined according to $v^{j}=\frac{u^{j}}{u^{0}}$, and the Lorentz factor, defined by $W \equiv u^{0}$, satisfies the familiar relation $W=\left(1-\mathrm{v}^{2}\right)^{-1 / 2}$, where $\mathrm{v}^{2}=\eta_{i j} v^{i} v^{j}$, with $\eta^{i j}$ being the spatial metric of Minkowski spacetime.

The components of the 5 -vector of conserved variables,

$$
\mathbf{F}^{0}(\mathbf{w})=\left(D, S^{j}, \tau\right)=\left(\rho W, \rho h W^{2} v^{j}, \rho h W^{2}-p-\rho W\right),
$$

are, respectively, the rest-mass density, the relativistic momentum density and the total energy density without the rest-mass density.

The three 5-vectors of fluxes are:

$$
\mathbf{F}^{i}(\mathbf{w})=\left(\rho W v^{i}, \rho h W^{2} v^{j} v^{i}+p \eta^{i j}, \rho h W^{2} v^{i}-\rho W v^{i}\right) .
$$

Assuming an EoS of the form $p=p(\rho, \varepsilon)$, the local sound velocity $c_{s}$ is given by

$$
h c_{s}^{2}=\chi+\left(\frac{p}{\rho^{2}}\right) \kappa, \quad \chi=\frac{\partial p}{\partial \rho}, \quad \kappa=\frac{\partial p}{\partial \epsilon} .
$$

Authors in [1] showed the characteristic structure of the multidimensional RHD system of equations. Some components of one righteigenvector, and also the determinant of the matrix of the righteigenvectors (Eqs. (17) and (21), respectively, in [1) contain the expression $\mathcal{K}:=\frac{\tilde{\kappa}}{\tilde{\kappa}-c_{s}^{2}}$, with $\tilde{\kappa}:=\frac{1}{\rho} \frac{\partial p}{\partial \epsilon}$. It is clear that $\mathcal{K}$ is not defined when $\tilde{\kappa}-c_{s}^{2}=0$, and therefore, in this case, the system of righteigenvectors given in [1] is not well behaved. Regarding this point, the following proposition can be proved.

Proposition The thermodynamic function $\tilde{\kappa}-c_{s}^{2}$ is identically zero iff $p=p_{o}(e), e:=$ $\rho(1+\epsilon)$, with $p_{o}$ being an arbitrary function.

Proof. 
$\tilde{\kappa}-c_{s}^{2}=0$, taking into account the definition of $c_{s}^{2}$, can be shown easily to be equivalent to $(1+\epsilon) \tilde{\kappa}-\chi=0$. Introducing the auxiliary variables $t:=\log (1+\epsilon)$ and $x:=\log \rho$, we conclude that $\tilde{\kappa}-c_{s}^{2}=0 \Leftrightarrow \frac{\partial p}{\partial t}-\frac{\partial p}{\partial x}=0$. The general solution of this advective-like partial differential equation (PDE) is $p=p_{o}(x+t)$, with $x+t=\log (\rho(1+\epsilon))=\log e$ and being $p_{o}$ an arbitrary function.

This conclusion can be also obtained in terms of functionals. $\tilde{\kappa}-c_{s}^{2}=0$, from the definitions of $c_{s}^{2}$ and $e$, is equivalent to $\frac{\partial e}{\partial \epsilon} \frac{\partial p}{\partial \rho}-\frac{\partial e}{\partial \rho} \frac{\partial p}{\partial \epsilon}=0$, which describes a functional dependence of $p$ and $e, p=p_{o}(e)$.

Hence, we have showed that $\tilde{\kappa}-c_{s}^{2}=0 \Leftrightarrow p=p_{o}(e)$.

From the above proposition, we conclude that the characteristic eigenvectors of the SRHD equations (5) derived in [1] have to be reviewed just in the case of barotropic EoS.

For the sake of conciseness, let us concentrate on the 1D version of the analysis presented in [1]. The expressions for the righteigenvectors are:

$$
\begin{aligned}
\mathbf{r}_{0} & =\left(\frac{\mathcal{K}}{h W}, v, 1-\frac{\mathcal{K}}{h W}\right), \\
\mathbf{r}_{ \pm} & =\left(1, h W\left(v \pm c_{s}\right), h W\left(1 \pm v c_{s}\right)-1\right) .
\end{aligned}
$$

Multiplying $\mathbf{r}_{0}$ by the normalization factor $\tilde{\kappa}-c_{s}^{2}$, the determinant of the matrix of renormalized righteigenvectors, $\Delta_{1 D}$, satisfies

$$
\Delta_{1 D}=\frac{2 c_{s}^{3} h}{W} .
$$

The singularity has been removed, both in the eigenvectors and the determinant, and the system of eigenvectors is complete for a general EoS, including a barotropic one.

Analogously, in the multidimensional case, it can be showed that, given an arbitrary spacelike 3 -vector $\zeta_{j}$, the general eigenvalue problem for the matrix $\zeta_{j} B^{j}$ (see equation (5) ) has the following renormalized righteigenvectors:

$$
\begin{aligned}
& \mathbf{r}_{01}=\left(\frac{\tilde{\kappa}}{h W},\left(\tilde{\kappa}-c_{s}^{2}\right) v^{i},\left(\tilde{\kappa}-c_{s}^{2}\right)-\frac{\tilde{\kappa}}{h W}\right), \\
& \mathbf{r}_{02,03}=\left(W v_{j} \bar{\zeta}^{j}, h\left(\bar{\zeta}^{i}+2 W^{2} v_{j} \bar{\zeta}^{j} v^{i}\right),(2 h W-1) W v_{j} \bar{\zeta}^{j}\right), \\
& \mathbf{r}_{ \pm}=\left(1, h W\left(\frac{\left(\mathcal{A}_{ \pm}-1\right)}{v_{j} \zeta^{j}} \zeta^{i}+v^{i}\right), h W \mathcal{A}_{ \pm}-1\right),
\end{aligned}
$$

where $\mathcal{A}_{ \pm}=\frac{\left(v_{j} \zeta^{j}\right)^{2}-\zeta_{j} \zeta^{j}}{\lambda_{ \pm} v_{j} \zeta^{j}-\zeta_{j} \zeta^{j}}$, and $\bar{\zeta}_{j}$ denotes the two unitary spacelike 3 -vectors which together with $\frac{\zeta_{j}}{\sqrt{\zeta_{j} \zeta^{j}}}$ form an orthonormal basis. The particular values of the above righteigenvectors in the direction $\zeta_{j}=(1,0,0)$ coincide, as it should be, with the ones derived in [1]. The singularity has been removed and all the components of the eigenvectors are well defined. The determinant of the righteigenvectors, $\Delta$, does not vanish,

$$
\Delta=\frac{-c_{s}^{2} W h^{3}\left(\lambda_{+}-\lambda_{-}\right)\left(\left(v_{j} \zeta^{j}\right)^{2}-\zeta_{j} \zeta^{j}\right)^{2}}{\left(\lambda_{+} v_{j} \zeta^{j}-\zeta_{j} \zeta^{j}\right)\left(\lambda_{-} v_{j} \zeta^{j}-\zeta_{j} \zeta^{j}\right) \sqrt{\zeta_{j} \zeta^{j}}} \neq 0
$$


and the system of eigenvectors is complete (even for a barotropic EoS).

Therefore, the main conclusion is that, by an appropriate renormalization of one of the eigenvectors derived in [1, it is possible to use the corresponding numerical hydrocode for a general EoS, including the case of barotropic EoS. Similar renormalization is required in RMHD when the characteristic eigenvalues of the system of equations become degenerate [17.

A second strategy is possible: to rewrite down the SRHD equations for a barotropic EoS. It is the one proposed in [5, 6, 7]. Yes, indeed, quoting Anile in [16] (page 11): 'Since the rest-mass density does not intervene in the state equation, these fluids can be described solely by the conservation of energy and momentum equations. The mass conservation equation decouples from the others and can be solved after the fluid motion has been determined'. The above Anile's quotation defines the (alternative) computational strategy to follow.

The conservation of momentum and energy equations governing the evolution of a barotropic fluid, i.e. the one satisfying a barotropic causal EoS, are

$$
\begin{aligned}
& \partial_{t}\left[(e+p) W^{2} v^{i}\right]+\partial_{j}\left[(e+p) W^{2} v^{i} v^{j}+p \eta^{i j}\right]=0, \\
& \partial_{t}\left[(e+p) W^{2}-p\right]+\partial_{j}\left[(e+p) W^{2} v^{j}\right]=0 .
\end{aligned}
$$

The new vector of conserved variables is $U=\left((e+p) W^{2} v^{i},(e+p) W^{2}-p\right)$. The system of equations (17) and (18) can be cast into the form of a quasi-linear system of PDEs:

$$
\partial_{t} U+A^{j} \partial_{j} U=0, \quad A^{j}=\left(\begin{array}{c|c}
A_{1}^{j} & A_{2}^{j} \\
\hline \delta_{k}^{j} & 0
\end{array}\right),
$$

where $A_{1}^{j}=v^{j} \delta_{k}^{i}+\left[\left(1-v^{2} c_{s}^{2}\right) \delta_{k}^{j} v^{i}+2 c_{s}^{2} v^{j} v_{k} v^{i}-2 c_{s}^{2} v_{k} \eta^{i j}\right]\left(1-v^{2} c_{s}^{2}\right)^{-1}$ and $A_{2}^{j}=$ $\left[-\left(1+c_{s}^{2}\right) v^{j} v^{i}+c_{s}^{2}\left(1+v^{2}\right) \eta^{i j}\right]\left(1-v^{2} c_{s}^{2}\right)^{-1}$.

Let $\zeta_{j}$ be an arbitrary spacelike vector. The eigenvalues of the matrix $\zeta_{j} A^{j}$ are

$$
\begin{aligned}
& \lambda_{0}=\zeta_{j} v^{j} \\
& \lambda_{ \pm}=\frac{\left(1-c_{s}^{2}\right) \zeta_{j} v^{j} \pm c_{s}\left(1-v^{2}\right)^{1 / 2}\left(\left(c_{s}^{2}-1\right)\left(\zeta_{j} v^{j}\right)^{2}+\left(1-v^{2} c_{s}^{2}\right) \zeta_{j} \zeta^{j}\right)^{1 / 2}}{1-v^{2} c_{s}^{2}}
\end{aligned}
$$

where $\lambda_{0}$ has multiplicity 2 . The corresponding eigenvalues associated with previous set of eigenvalues are

$$
\begin{aligned}
& r_{0}=\left(\begin{array}{c}
\left(1-v^{2}\right) \bar{\zeta}^{i}+2 \bar{\zeta}_{j} v^{j} v^{i} \\
2 \bar{\zeta}_{j} v^{j}
\end{array}\right), \\
& r_{ \pm}=\left(\begin{array}{c}
\left(\zeta_{j} v^{j}-\lambda_{ \pm}\right) \zeta^{i}+\left(\lambda_{ \pm} \zeta_{j} v^{j}-\zeta_{j} \zeta^{j}\right) v^{i} \\
\left(\zeta_{j} v^{j}\right)^{2}-\zeta_{j} \zeta^{j}
\end{array}\right),
\end{aligned}
$$

where $\bar{\zeta}_{j}$ denotes, as previously, the two unitary spacelike 3 -vectors which together with $\frac{\zeta_{j}}{\sqrt{\zeta_{j} \zeta^{j}}}$ form an orthonormal basis. The system of eigenvalues form a complete basis. Therefore, the system of equations (19) is hyperbolic.

In brief, according to this second strategy and for a barotropic EoS, the numerical procedure, regarding the use of Godunov-type methods, would consist in solving, first, 
the system (19). The rest-mass density can be obtained, in a second step, by solving the continuity equation in the field velocity provided by the first step. Quoting Anile in [16] (page 11): '...the only role of the continuity equation is to provide a volume tracer.'

\section{Summary}

The characteristic formulation of the RHD equations carried out in [1] needs a minor modification for a barotropic EoS in order to remove the singularity in one of the eigenvectors.

Any user of a relativistic hydro-code, based on Godunov-type techniques and designed for a general $\operatorname{EoS} p=p(\rho, \epsilon)$, that might be interested in using a barotropic EoS, can follow one of the following two strategies. (A) Just to renormalize one of the eigenvectors, such as we have shown above. (B) To rewrite explicitly the SRHD equations for a barotropic EoS.

In terms of efficiency, it is obvious that strategy (B) is the most recommendable, since there is one equation less in the system. However, we recommend strategy (A) due to the fact that it can be applied in studies of flows obeying a general EoS, including the barotropic ones. Moreover, strategy (A) allows one to handle with those flows that, during their evolution, might change their thermodynamical properties in such a way that the EoS admits a barotropic branch as, e.g., the asymptotic behavior of the EoS

for dense matter at high densities. In such a case, strategy (A) would be the only one suitable for a global description of the evolution of these flows.

\section{Acknowledgments}

Work partially supported by the Spanish Ministry of Science (grants: AYA2010-21097C03-01 and AYA2010-21097-C03-2). I. C.-C. acknowledges support from Alexander von Humboldt Foundation.

\section{References}

[1] Donat R, Font J A, Ibáñez J M and Marquina A 1998 J. Comput. Phys. 14658

[2] Peacok J A 1999 Cosmological Physics (Cambridge, England: Cambridge University Press)

[3] Camenzind M 2007 Compact Objects in Astrophysics: White Dwarfs, Neutron Stars and Black Holes (Berlin, Germany: Springer-Verlag)

[4] Smoller J, Temple B 1993 Commun. Math. Phys. 15667

[5] Mach P 2010 Phys. Rev E 81046313

[6] Mach P 2011 arXiv:1104.3751v1 [math-ph] 19 Apr 2011

[7] Ryu D, Chattopadhyay I, Jang H 2011 Relativistic Hydrodynamic Codes for Adiabatic and Isothermal Flows, in Numerical Modeling of Space Plasma Flows (ASTRONUM-2010), ASP Conference Series, 444. Eds: N. Pogorelov, E. Audit and G. Zank (San Francisco: Astronomical Society of the Pacific)

[8] Martí J M, Ibáñez J M and Miralles J A 1991 Phys. Rev. D 433794

[9] Font J A, Ibáñez J M, Martí J M and Marquina A 1994 Astron. Astrophys. 282304

[10] Banyuls F, Font J A, Ibáñez J M, Martí J M and Miralles J A 1997 Astrophys. J. 476221 
[11] Ibáñez J M, Aloy M A, Font J A, Martí J M, Miralles J A and Pons J A 2001 Proc. from the Int. Conf. Godunov Methods: Theory and Applications (Oxford, United Kingdom) (New York: Kluwer Academic/Plenum Publishers, Ed. E. F. Toro)

[12] Martí J M and Müller E 2003 Living Rev. Relativity 6 http://www.livingreviews.org/lrr-2003-7

[13] Font J A 2008 Living Rev. Relativity 11 http://www.livingreviews.org/lrr-2008-7

[14] LeVeque R J 1992 Numerical Methods for Conservation Laws (Basel, Switzerland: Birkhaüser)

[15] Toro E F 2009 Riemann solvers and numerical methods for fluid dynamics: a practical introduction (Berlin, Germany: Springer, 3rd. edition)

[16] Anile M A 1989 Relativistic fluids and magneto-fluids (Cambridge, England: Cambridge University Press)

[17] Antón L, Miralles J A, Martí J M, Ibáñez J M, Aloy M A and Mimica P 2010 Astrophys. J. Suppl. 1871 